\title{
Glucose fluctuation increased hepatocyte apoptosis under lipotoxicity and the involvement of mitochondrial permeability transition opening
}

\author{
Xueyao Yin", Fenping Zheng ", Qianqian Pan, Saifei Zhang, Dan Yu, Zhiye Xu and Hong Li \\ Department of Endocrinology, School of Medicine, The Affiliated Sir Run Run Shaw Hospital, \\ Zhejiang University, 3 East Qingchun Road, Hangzhou 310016, China \\ * $(X$ Yin and $F$ Zheng contributed equally to this work)
}

Correspondence should be addressed to $\mathrm{H} \mathrm{Li}$

Email

lihongheyi@126.com

\begin{abstract}
Oxidative stress is considered to be an important factor in producing lethal hepatocyte injury associated with nonalcoholic fatty liver disease (NAFLD). Glucose fluctuation, more pronounced in patients with diabetes, has been recognized as an even stronger oxidative stress inducer than the sustained hyperglycemia. Here, we investigated the role of glucose variability in the development of the NAFLD based on hepatocyte apoptosis and possible mechanisms. To achieve this goal we studied C57BL/6J mice that were maintained on a high fat diet (HFD) and injected with glucose ( $3 \mathrm{~g} / \mathrm{kg}$ ) twice daily to induce intermittent high glucose (IHG). We also studied hepatic L02 cells incubated with palmitic acid (PA) to induce steatosis. The following experimental groups were compared: normal glucose (NG), sustained high glucose (SHG) and IHG with or without PA. We found that, although hepatic enzyme levels and liver lipid deposition were comparable between HFD mice injected with glucose or saline, the glucose injected mice displayed marked hepatocyte apoptosis and inflammation, accompanied by increased lipid peroxide in liver. In vitro, in the presence of PA, IHG increased L02 cell apoptosis and oxidative stress and produced pronounced mitochondrial dysfunction relative to the NG and SHG groups. Furthermore, treatment with the mitochondrial permeability transition (MPT) inhibitor, cyclosporin A $(1.5 \mu \mathrm{mol} / \mathrm{l})$, prevented mitochondrial dysfunction, oxidative stress and hepatocyte apoptosis. Our data suggests that IHG under lipotoxicity might contribute to the development of NAFLD by increasing oxidative stress and hepatocyte apoptosis via MPT and its related mitochondrial dysfunction.
\end{abstract}

Key Words

- glucose fluctuation

- nonalcoholic fatty liver disease

- apoptosis

mitochondrial permeability transition

\section{Introduction}

Nonalcoholic fatty liver disease (NAFLD) consists of ectopic fat accumulation in the liver secondary to metabolic factors, mostly obesity and insulin resistance (IR) or diabetes mellitus (Adams \& Angulo 2005). The prevalence of NAFLD has increased dramatically in recent years and affects $15-30 \%$ of the general population (Bellentani \& Marino 2009). However, in individuals with type 2 diabetes (T2DM), the prevalence of NAFLD is 
up to $70 \%$ (Targher et al. 2007, Leite et al. 2009, Prashanth et al. 2009).

The spectrum of NAFLD ranges from simple steatosis in its most benign form to cirrhosis on the opposite end of the spectrum where most liver-related morbidity and mortality occur. Nonalcoholic steatohepatitis (NASH) is a lesion of intermediate severity, which is accompanied by hepatocyte injury and death, as well as hepatic infiltration of inflammatory cells. NASH-related liver damage often triggers liver fibrosis. Studies have shown that fibrosis progresses in $38 \%$ of patients with NAFLD over a mean follow-up interval of 5.3 years and the presence of necroinflammation on initial biopsy is the strongest predictor of progression (Argo et al. 2009). The 'two-hit' hypothesis is a widely accepted paradigm to explain the progression of NAFLD (Day \& James 1998). The first hit is the development of hepatic steatosis, and the second hit includes oxidative, metabolic and cytokine stresses that overwhelm hepatocyte survival mechanisms, leading to hepatocyte death. Furthermore, some authors consider hepatocyte apoptosis as a third hit that promotes the development of cirrhosis (Jou et al. 2008).

Although the mechanisms underlying NAFLD development have not yet been clearly elucidated, mitochondrial dysfunction has been shown to play a critical role in triggering hepatocyte apoptosis (Malhi \& Gores 2008). Mitochondria burns excessive intrahepatic fat by $\beta$-oxidation. However, this reaction produces not only ATP but also reactive oxygen species (ROS). An increase in ROS reaching a threshold level can trigger the opening of the mitochondrial permeability transition (MPT) pore, which in turn leads to the simultaneous collapse of mitochondrial membrane potential $(\Delta \psi)$ and a massive release of ROS (Zorov et al. 2000). The MPT is defined as a rapid increase in the permeability of the mitochondrial membrane to low molecular solutes (Lemasters et al. 1998). The opening of the MPT pore can trigger numerous subsequent reactions, for example, mitochondrial release of cytochrome c into the cytosol triggers the assembly of the caspase 9-caspase 3 activation complex (Russmann et al. 2009). It has also been shown that inhibiting the MPT with specific blockers, such as cyclosporin A (CsA), ameliorates caspase activation and apoptosis in several cellular systems (Halestrap et al. 1997, Kroemer \& Reed 2000, Halestrap \& Brenner 2003).

Recently, it has been shown that the glycemic variability is an independent predictive factor for the progression of NAFLD in a diabetic population (Hashiba et al. 2013). Mechanistic studies have indicated that chronic sustained hyperglycemia is associated with increased mitochondrial ROS production, which was further exacerbated by conditions of intermittent high glucose (IHG) (Quagliaro et al. 2003, 2005). Clinical studies have also shown that various markers of oxidative damage are significantly related to the mean amplitude of glycemic excursions (Monnier et al. 2006, Zheng et al. 2010, Wang et al. 2011). Despite this association between glycemic variability and ROS production, no systematic experiments have been conducted to elucidate the role of glucose fluctuations in ROS production and apoptosis in the liver. Furthermore, the contribution of glucose fluctuation to the development of NAFLD in T2DM remains to be elucidated. With this in mind, the aim of the current study was to examine the effects of IHG on the development of NAFLD. Moreover, the possible involvement of MPT pore opening was investigated.

\section{Materials and methods}

\section{Animal experiments and treatment}

Four-week-old male C57BL/6J mice were purchased from the Slack Experimental Animal Center of Chinese Academy of Sciences (Shanghai, China) and fed a standard diet (STD; $70 \%$ carbohydrate, $20 \%$ protein and $10 \%$ fat). After 1 week of habituation, animals were weighed and divided into two groups: the experimental group $(n=24)$ was fed a high fat diet (HFD; $35 \%$ carbohydrate, $20 \%$ protein and $45 \%$ fat), and the control group $(n=12)$ was maintained on a STD. After 8 weeks, the experimental group was further randomized into two groups of 12 mice. One group (HFD +F group) was treated with $3 \mathrm{~g} / \mathrm{kg}$ glucose by i.p. injection twice daily (at 0800 and $1600 \mathrm{~h}$ ), while the remaining experimental animals $(\mathrm{HFD}+\mathrm{NS})$ and the control group $(\mathrm{STD}+\mathrm{NS})$ were injected with saline. All mice were weighed daily, and their fasting blood glucose levels were measured using One Touch Ultra glucose strips (LifeScan, Milpitas, CA, USA) twice monthly, and fasting insulin levels were measured at 8 and 20 weeks of HFD using an insulin EIA kit (Millipore, Billerica, MA, USA). The animal care committee of Zhejiang University approved all animal experiments.

\section{Biochemical analyses of blood and liver samples}

Free fatty acid (FFA) levels and alanine aminotransferase (ALT) activity in the fasted serum were measured using an FFA assay kit (Cayman Chemical, Ann Arbor, MI, USA) and ALT activity assay kit (Bivision, Mountain View, CA, USA) respectively. The concentration of ATP and triglycerides in the liver was determined using an ATP colorimetric assay

Published by Bioscientifica Ltd. 
kit (Bivision) and a triglyceride quantification colorimetric kit (Bivision) respectively.

\section{Histopathology}

Liver tissue was collected from all mice and processed using standard methods. Paraffin-embedded sections of the liver were stained with hematoxylin and eosin (H\&E). Frozen liver sections were stained with Oil Red $\mathrm{O}$ to assess hepatic lipid content. Immunohistochemical staining was carried out to determine the levels of 4-hydroxy-2-nonenal (HNE; 1:100, rabbit anti-mouse; 1:500; Abcam, Cambridge, MA, USA), malondialdehyde (MDA; 1:250, rabbit anti-mouse; 1:500; Abcam) and $\alpha$-smooth-muscle-actin ( $\alpha$-SMA; 1:100; Epitomics, Burlingame, CA, USA; rabbit anti-mouse; 1:500) using standard protocols. The extent of hepatocyte apoptosis in the liver was determined using a TUNEL kit (Roche Diagnostics, Indianapolis, IN, USA). To quantify apoptosis, ten visual fields at $400 \times$ from each section were analyzed for TUNEL-positive cells. A TUNEL index was determined using the following formula: (number of stained cells/total number of cells) $\times 100$ (Yamada et al. 2007).

\section{Western blotting}

Western blotting was performed according to standard methods, using equal amounts of protein $(50 \mu \mathrm{g})$. Primary antibodies raised against HNE and MDA (Abcam), Bax, cleaved caspase 9, cleaved caspase 3, cleaved PARP, CCAAT/-enhancer-binding protein homologous protein (CHOP), p53 up-regulated modulator of apoptosis (PUMA), cytochrome c, $\beta$-actin (all 1:1000; Cell Signaling Technology, Beverly, MA, USA) and $\alpha$-SMA (1:1000; Epitomics) were used. Immunoreactive proteins were detected using a chemiluminescent ECL assay kit (Millipore).

\section{Real-time quantitative RT PCR}

A standard real-time quantitative RT PCR (qRT-PCR) was performed. Total RNA was extracted from tissues using TRIzol (Invitrogen, Carlsbad, CA, USA). The mRNA levels were assessed by real-time qRT-PCR using the SYBR Green PCR Master Mix kit (TaKaRa Bio, Inc., Shiga, Japan) with a LightCycler 480 II system (Roche Applied Science). Primer pairs for transcripts of interest are listed in Supplementary Table S1, see section on supplementary data given at the end of this article. GAPDH was chosen as an invariant standard. All experimental tissues and standard curve samples were run in duplicate in a 96-well reaction plate (MicroAmp Optical, Applied Biosystems). Results are expressed as fold expression relative to expression in the control group following the $\Delta \Delta C \mathrm{t}$ method (Livak \& Schmittgen 2001).

\section{Hepatocyte culture and treatment}

L02 cells are a normal hepatocyte cell line derived from adult liver tissue. These cells were obtained from the Cell Bank of Type Culture Collection of the Chinese Academy of Science (Shanghai, China) and were maintained in RPMI 1640 medium containing 10\% fetal bovine serum. Palmitic acid (PA) conjugated-BSA was prepared as described previously (Shi et al. 2006). Cells incubated with or without PA $(0.125 \mathrm{mmol} / \mathrm{l})$ were exposed to normal glucose (NG, $5.5 \mathrm{mmol} / \mathrm{l})$, sustained high glucose (SHG, $33.3 \mathrm{mmol} / \mathrm{l}$ ) or IHG (5.5 and $33.3 \mathrm{mmol} / \mathrm{l}$ alternating every $12 \mathrm{~h}$ ) for 3 days. To assess MPT, cells were pre-incubated overnight with the MPT blocker CsA $(1.5 \mu \mathrm{mol} / \mathrm{l})$ or the appropriate vehicle (DMSO).

\section{Annexin V staining for apoptosis detection}

To assess apoptosis in L02 cells, the level of Annexin V staining was determined by flow cytometry. After incubation, cells were treated according to the manufacturer's protocol (FITC Annexin V Apoptosis Detection Kit, BD Biosciences Pharmingen, San Diego, CA, USA) and analyzed by flow cytometry (FACSort, Becton Dickinson, Franklin Lakes, NJ, USA).

\section{Measurement of ROS generation}

Aliquots of L02 cells were removed at timed intervals and analyzed for the generation of total ROS using the fluorescent probe dichlorodihydrofluorescein diacetate (DCF/DA, Beyotime, Haimen, Jiangsu, China) by flow cytometry at $480 \mathrm{~nm}$ excitation and $540 \mathrm{~nm}$ emission settings.

\section{Mitochondrial superoxide assay}

Mitochondrial superoxide levels were measured by flow cytometry after staining with the mitochondrial superoxide-specific dye MitoSOX red (Molecular Probes, Eugene, OR, USA) at $396 \mathrm{~nm}$ excitation and 580/510 nm emission settings.

\section{Measurement of the mitochondrial membrane potential}

The $\Delta \psi$ was estimated by measuring the fluorescence of 5, 5', 6, 6'-tetrachloro-1, 1', 3, 3'-tetraethylbenzimidazolcarbocyanine iodide (JC-1, Beyotime) by flow cytometry.

Published by Bioscientifica Ltd 
At relatively low concentrations, JC- 1 exists as a monomer that fluoresces at $527 \mathrm{~nm}$, and when concentrated by actively respiring mitochondria, JC-1 forms aggregates, which fluoresce at $590 \mathrm{~nm}$ (Salvioli et al. 1997). The intensity of fluorescence at $590 \mathrm{~nm}$ is proportional to the $\Delta \psi$, which indicates a closed MPT pore. On induction of MPT, dissipation of the $\Delta \psi$ prevents the formation of JC-1 aggregates with reduced fluorescence at $590 \mathrm{~nm}$.

\section{Electron microscopy}

For electron microscopy, the specimens were fixed and embedded according to standard protocols. Embedded specimens were cut into ultrathin sections of $0.5 \mu \mathrm{m}$ and stained with uranyl acetate and lead citrate. The sections were then observed under an Hitachi H-7100 transmission electron microscope (Hitachi-High Technologies Co., Shimbashi, Tokyo, Japan).

\section{Statistical analysis}

All data have been expressed in terms of means \pm s.E.M. Differences between the means of individual groups were assessed with the independent $t$-test or a one-way ANOVA and false discovery rate (FDR) multiple range tests. All statistical analysis was performed using SPSS 20.0 (IBM, Armonk, NY, USA). A significant difference was defined as $P<0.05$.

\section{Results}

Effect of glucose fluctuation on body weight, metabolic parameters and hepatic function

Blood glucose levels were measured to test the amplitude of fluctuations after a bolus of i.p. glucose injection. The mice fed a HFD and given an i.p. glucose injection showed significant fluctuations as compared to those given an i.p. saline injection and fed on a HFD or STD (Supplementary Figure S1, see section on supplementary data given at the end of this article). Mice fed a HFD were significantly heavier than those fed a STD during the 5-month experimental time period $(P<0.05)$, whereas there was no difference in body weight between HFD mice given glucose or saline injections ( $P>0.05$, Fig. $1 \mathrm{~A})$.

Fasting blood glucose levels were significantly higher in mice fed a HFD compared to mice fed a STD at 6 weeks $(P<0.01)$, and the difference was maintained throughout the rest of the experiment (Fig. 1B). There was no difference in fasting blood glucose levels between HFD mice given glucose or saline injections $(P>0.05)$. Similarly, fasting insulin levels were greatly increased in HFD fed mice $(P<0.05$ at 8 weeks and $P<0.01$ at 20 weeks), with no differences of the levels between HFD mice receiving glucose or saline injections (Fig. 1C).

Elevated circulating FFA is an established risk factor for NAFLD (Unger \& Orci 2002, Feldstein et al. 2004). Serum FFA levels in HFD mice were significantly higher than those in STD mice at 8 weeks and were further increased

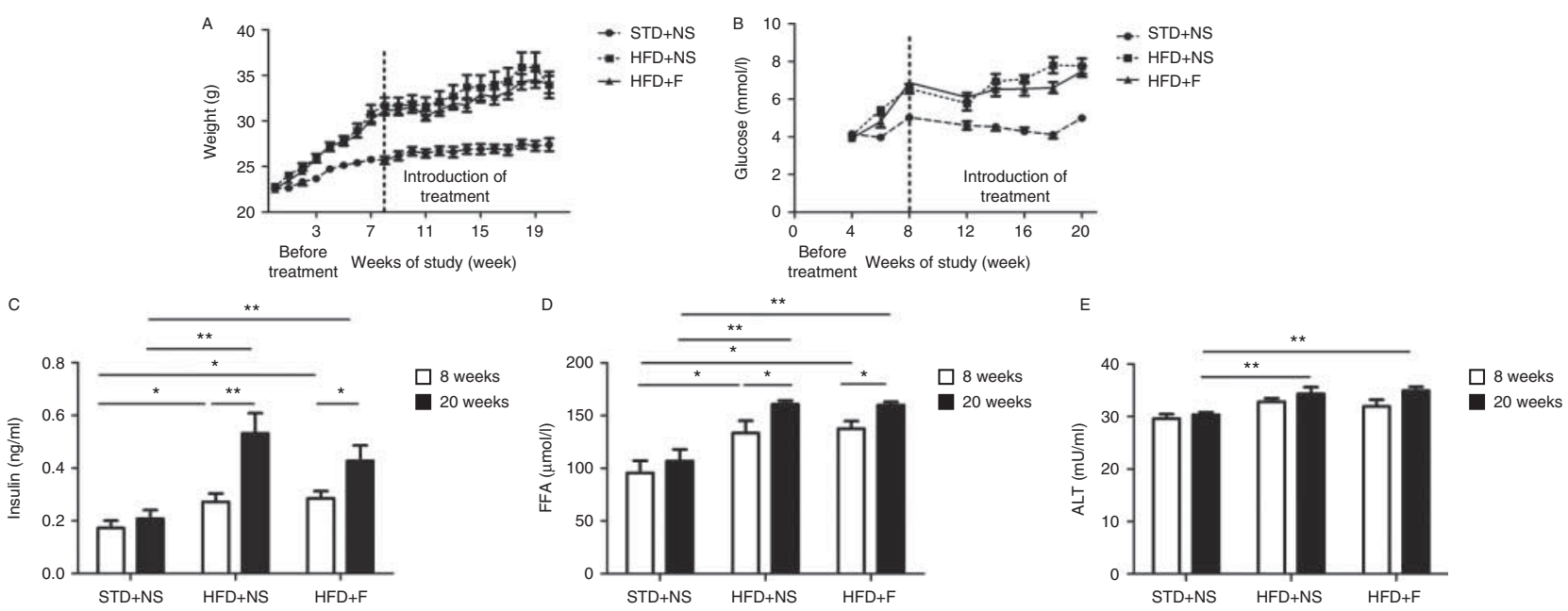

Figure 1

Effect of glucose fluctuation on body weight, metabolic parameters and hepatic function. Body weight $(A)$ and blood glucose levels $(B)$ were measured throughout the experimental time course. At weeks 8 and 20, fasting insulin, free fatty acid (FFA) and alanine aminotransferase (ALT) (C, D, E, respectively) levels were measured. Values are means \pm S.E.M.; $n=10-12$ mice/group; ${ }^{*} P<0.05, * * P<0.01$. 
at 20 weeks. FFA levels were not different between the HFD mice given saline or glucose injection at 8 or 20 weeks (Fig. 1D). At 8 weeks, the plasma ALT level was similar between STD and HFD mice, whereas a significant increase in plasma ALT was observed at 20 weeks $(P<0.01$, Fig. $1 \mathrm{E})$. No difference in the ALT level was found between the HFD mice given saline or glucose injection.

\section{Effect of blood glucose fluctuation on hepatic steatosis}

H\&E staining revealed significant vacuoles present in the liver of mice fed a HFD, which was also evident in Oil Red O stained sections, suggesting these mice had developed hepatic steatosis; however, IHG did not seem to exacerbate this condition (Fig. 2A and B). Consistently, the hepatic triglyceride levels in HFD mice were significantly higher than STD mice $(P<0.01)$, with no difference between HFD mice given saline or glucose injections (Fig. 2C).

\section{Glucose fluctuation increases hepatic apoptosis in vivo and vitro}

Emerging data has indicated that hepatocyte apoptosis is a histological hallmark that differentiates simple steatosis from NASH; we thus investigated the effect of IHG on hepatocyte apoptosis. The percentage of TUNEL-positive stained cells was low in STD mice; however, it was significantly higher in HFD mice given saline injections and further elevated in HFD mice given glucose injections $(P<0.01$, Fig. 3A).

A
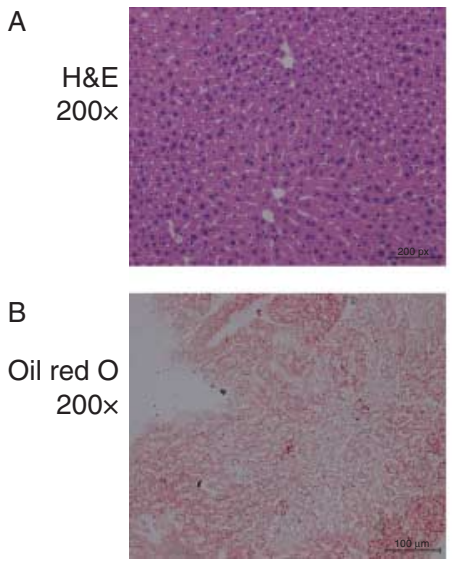

STD+NS
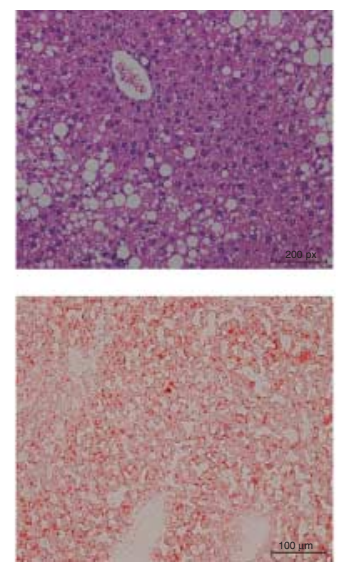

HFD+NS
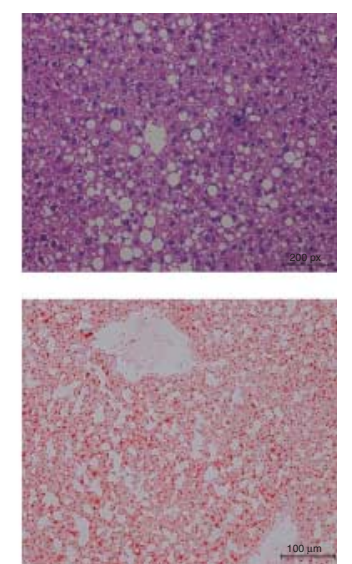

$\mathrm{HFD}+\mathrm{F}$
To investigate the molecular mechanism through which IHG induces hepatocyte apoptosis, we studied this phenomenon in L02 cells. First we confirmed the effect of IHG on hepatocytes in vitro. In the presence of PA, treatment with SHG-induced apoptosis relative to the NG group $(P<0.05$ vs $N G+P$ group). This effect was more pronounced in cells treated with IHG, which had a higher rate of apoptosis than SHG-treated cells $(P<0.05$ vs SHG + P group; Fig. 3B). There was no significant effect of SHG or IHG on hepatocyte apoptosis in the absence of PA.

PARP-specific proteolytic cleavage by caspases has been recognized as a characteristic of apoptosis, thus we investigated the cleavage PARP and caspases levels by western blotting. We observed that apoptosis-related proteins were significantly increased when L02 cells were incubated with PA and SHG relative to those with $\mathrm{PA}$ and NG treatment and that these proteins were further increased by treatment with PA and IHG (Fig. 3C). Similarly, cleaved caspase 3 , cleaved caspase 9 , Bax, CHOP and PUMA were also significantly increased in the liver of mice fed a HFD, which was further exacerbated by glucose injections (Fig. 3D).

\section{Glucose fluctuation exacerbates liver inflammation and fibrosis}

The intensity of the reparative process generally parallels with the degree of hepatocyte death, resulting in a variable distortion of the hepatic architecture with immune cells infiltration, fibrosis, and epithelial nodules regeneration

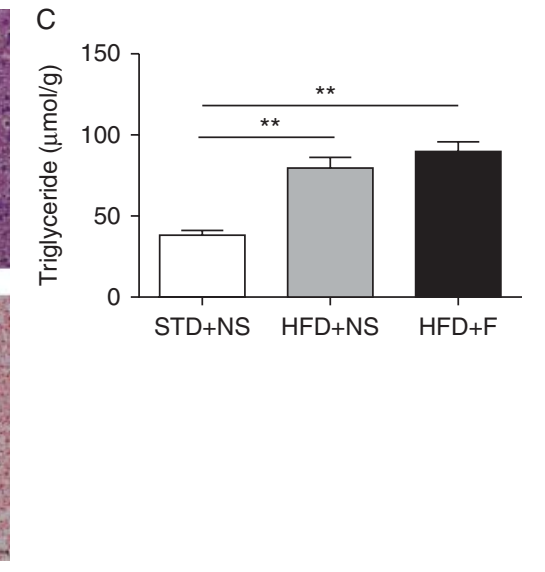

Red O (B) are shown. Scale bars, $100 \mu \mathrm{m}$. Hepatic triglyceride contents (C) are also shown. Values are means \pm s.E.M.; $n=10-12$ mice/group; $* * P<0.01$.

Published by Bioscientifica Ltd.
C) 2015 Society for Endocrinology Printed in Great Britain 

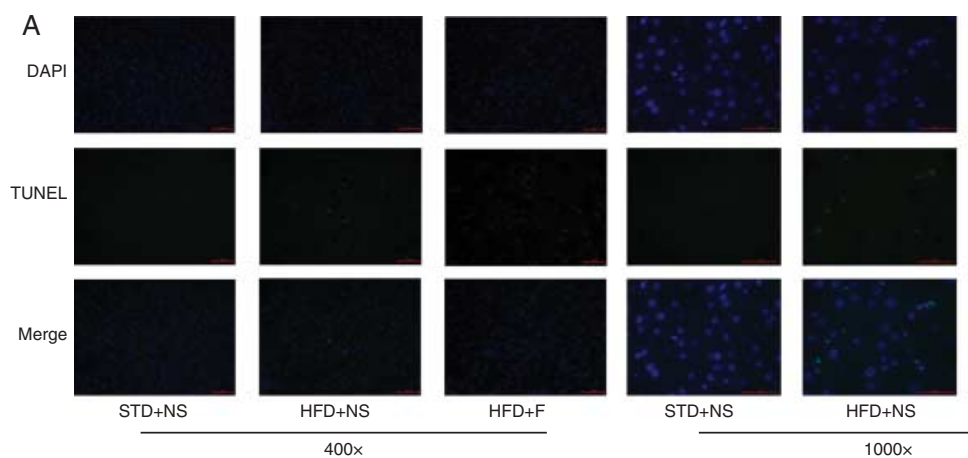

STD+NS

B

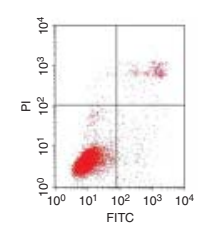

NG

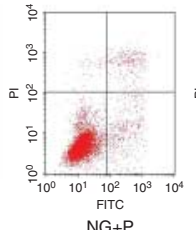

$\mathrm{NG}+\mathrm{P}$

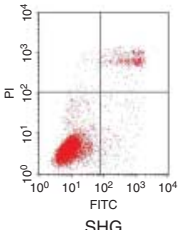

SHG

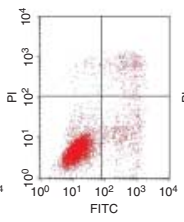

SHG+P

C

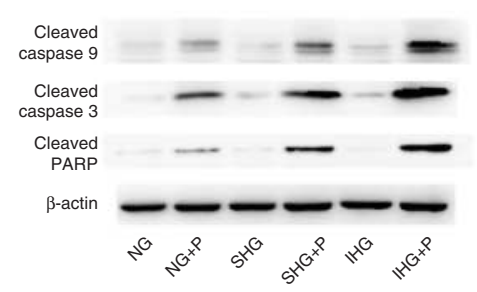

Figure 3

Effect of glucose fluctuation on hepatocyte apoptosis in vivo and vitro. Representative images of liver sections stained with TUNEL are presented and the percentage of TUNEL-positive cells quantified. Scale bars, $100 \mu \mathrm{m}$ (A). Green: TUNEL-positive cells; blue: DAPI stained nuclei. Representative data obtained by Annexin $V$ staining and quantification of the number of

(Jou et al. 2008). Therefore, we investigated the production of inflammatory markers in vivo. Although there was no difference in the hepatic expression of F4/80 (Emr1) mRNA among all groups of mice, we noted a significant increase in the mRNA levels of MCP-1 $(P<0.05)$ in the liver of mice fed a HFD, with the highest mRNA levels of MCP-1 and TNF- $\alpha$ found in the liver of HFD mice exposed to IHG (Fig. 4A).

We further examined the levels of liver fibrosis. The mRNA levels of ECM components, such as type 1 procollagen $\alpha 1$ (Col1a1) and type 4 procollagen $\alpha 1$ (Col4a1), fibrosis markers, such as transforming growth factor beta 1 (TGFb1), platelet-derived growth factor receptor (PDGFR) and tissue inhibitor of metalloprotease-1 (TIMP1), were
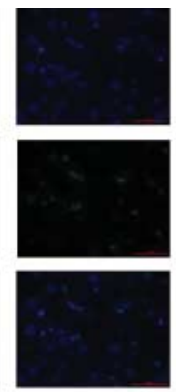

$1000 x$
D

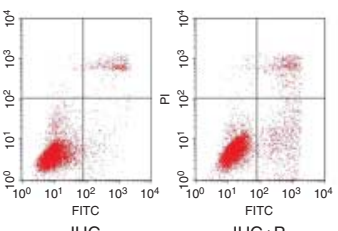

IHG

$\mathrm{IHG}+\mathrm{P}$

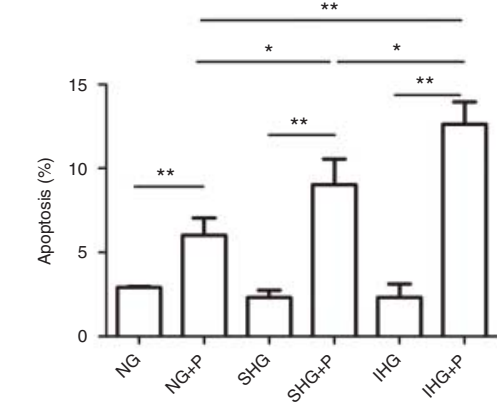

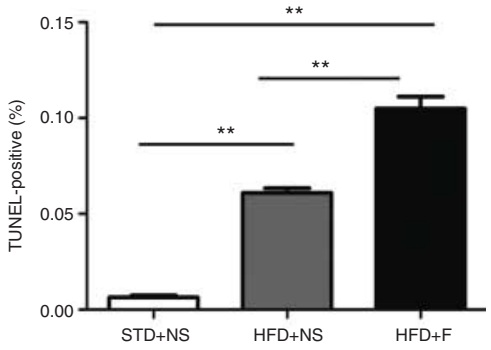

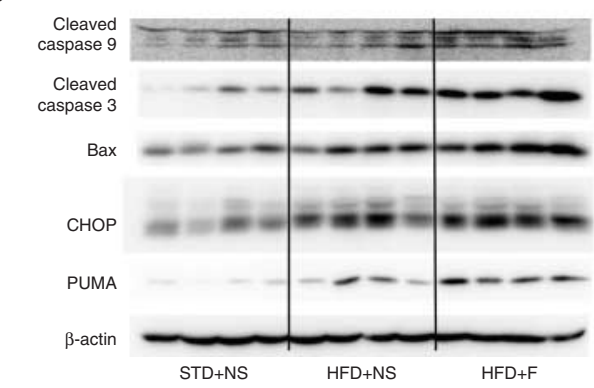

apoptotic cells by flow cytometry is presented (B); ${ }^{*} P<0.05, * * P<0.01$. Values are mean \pm S.E.M. of three replicates in three separate experiments. Representative western blots are presented showing the protein expression level of apoptosis-related proteins in L02 cells (C) and mice (D).

significantly increased in HFD-fed mouse livers, and the mRNA levels of Col1a1, Col4a1, TGFb1 and TIMP1 were even higher in mice exposed to IHG (Fig. 4B). This is in agreement with those obtained by western blotting (Fig. 4C) and immunohistochemistry (Fig. 4D) of $\alpha$-SMA expression.

\section{Blood glucose fluctuation promotes hepatic oxidative stress}

Several studies on the pathogenesis of NASH have highlighted the central role of excessive activation of mitochondrial oxidative stress. To address this issue, we examined the effect of IHG on oxidative stress in vivo and

Published by Bioscientifica Ltd 

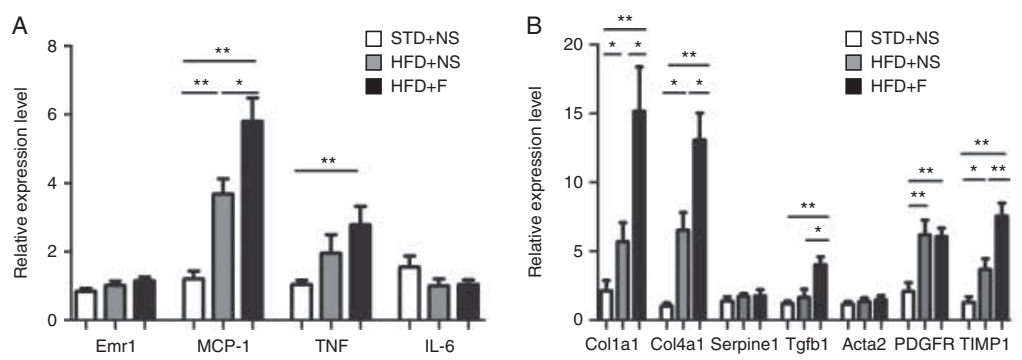

C
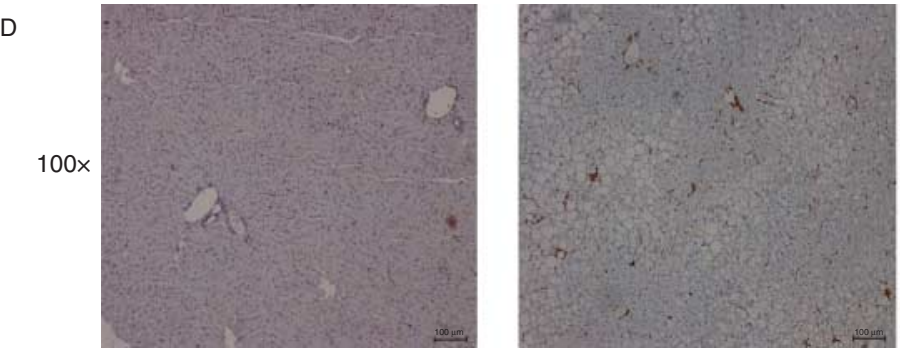

HFD+NS

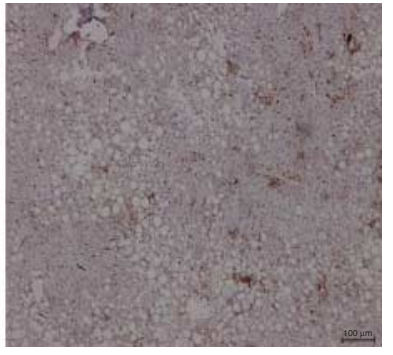

$\mathrm{HFD}+\mathrm{F}$

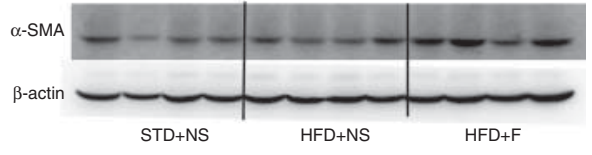

Figure 4

Effect of glucose fluctuation on liver inflammation and fibrosis. mRNA levels were determined by real-time $\mathrm{qRT}$-PCR, values are means \pm s.E.M.; $n=10-12$ mice/group; $* P<0.05, * * P<0.01$ (A and B). Representative

(C) in mice (C). Representative images of immunostained liver sections showing the expression of $\alpha$-SMA (D) are presented.

in vitro. L02 cells exposed to PA and SHG produced increased levels of ROS and mitochondrial superoxide relative to cells treated with PA and NG $(P<0.05)$, with even higher ROS and mitochondrial superoxide levels detected in cells treated with IHG with PA $(P<0.05$ vs SHG + P group; Fig. 5A and B). In the absence of PA, SHG or IHG had no effect on oxidative stress in L02 cells.

In vivo, ROS production can increase lipid peroxidation, leading to the production of HNE and MDA. To assess the effect of IHG on oxidative stress in mice fed a HFD, we assessed the abundance of HNE and MDA in the liver. Our immunohistochemistry (Fig. 5C and D) and western blotting data (Fig. 5E) both indicated that after 20 weeks of consuming a HFD, the HNE and MDA levels were significantly increased in the liver and these levels were further increased in mice exposed to IHG.

\section{Blood glucose fluctuation induced mitochondrial dysfunction by affecting the MPT}

To assess the effect of IHG on mitochondria, we studied various markers of mitochondrial function. Excess ROS production in mitochondria can trigger the MPT, resulting in impairment of mitochondrial function with effects on cellular metabolism and apoptosis. We therefore examined whether increased ROS production induced by IHG in the presence of PA was associated with mitochondrial dysfunction. Fluorescence of JC-1 aggregates was significantly reduced in L02 hepatocytes exposed to SHG with PA $(P<0.05$ vs NG $+P$ group $)$ and was further decreased in cells exposed to IHG and PA ( $P<0.05$ vs SHG + P group; Fig. 6 A). In the absence of $\mathrm{PA}$, the different glucose treatments had no effect on mitochondrial $\Delta \psi$. Mitochondrial morphology was subsequently assessed by electron microscopy. This data revealed that the mitochondria of cells treated only with NG had normal cristae, whereas there were reduced cristae and mitochondrial swelling in cells exposed to SHG and IHG, which was exacerbated by exposure to PA. The combined effect of PA and SHG led to more translucide matrix with less cristae formation than PA and NG and cells treated with IHG and PA showing an even greater effect (Fig. 6B). Cytochrome c release from mitochondria involved apoptosis, therefore cytoplasmic cytochrome c levels were assessed. L02 cells exposed to PA and IHG had the highest levels of cytochrome c among all the groups (Fig. 6C). In agreement with this observation, mice fed a HFD and injected with glucose also had the highest release of cytochrome c (Fig. 6D). Finally, we measured hepatic ATP content as another index of mitochondrial function. Here, we found that mice fed a HFD and injected with saline had higher hepatic levels of ATP compared to mice fed a STD $(P<0.01)$; however, mice fed a HFD and injected with glucose had a significantly lower level of ATP ( $P<0.05$ vs HFD + NS group), indicating impaired ATP production (Fig. 6E). 


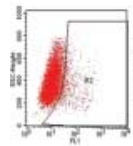

NG

B

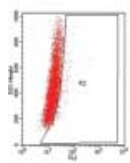

$N G$
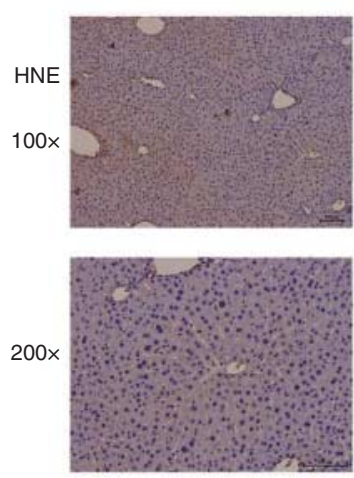

STD+NS

D
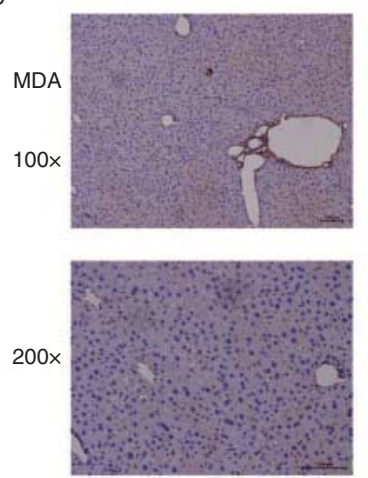

STD+NS

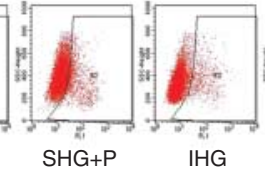

IHG

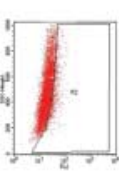

$\mathrm{SHG}+\mathrm{P}$

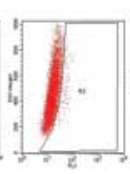

IHG
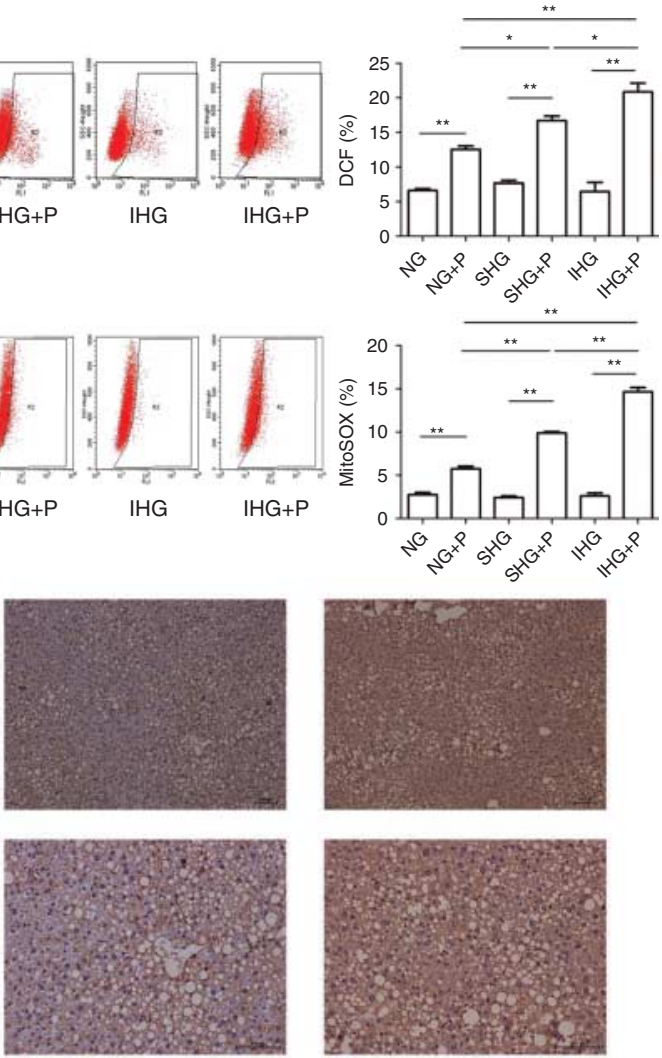

HFD+NS

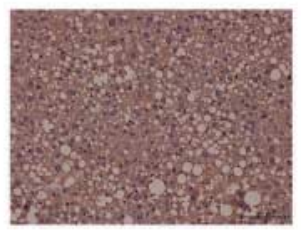

HFD+F
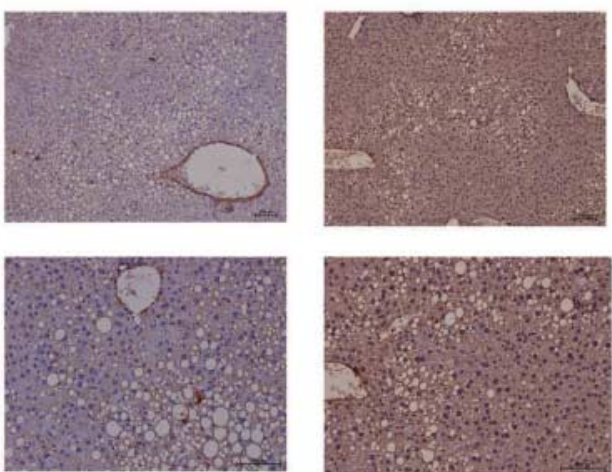

HFD+NS

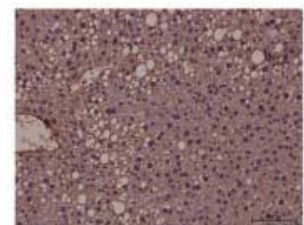

$\mathrm{HFD}+\mathrm{F}$

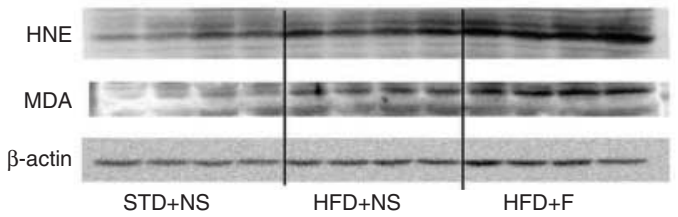

Figure 5

Effect of glucose fluctuation on hepatic oxidative stress. Representative histograms of flow cytometry showing dichlorodihydrofluorescein diacetate (DCF/DA) fluorescence (A) and MitoSOX Red fluorescence (B);

${ }^{\star} P<0.05,{ }^{*} P<0.01$; values are mean \pm S.E.M. of three replicates from three separate experiments. Representative images of immunostained liver sections showing the expression of HNE (C) and MDA (D) are presented. Scale bars, $100 \mu \mathrm{m}$. Representative western blots are also presented showing the protein expression level of HNE and MDA (E). 
A
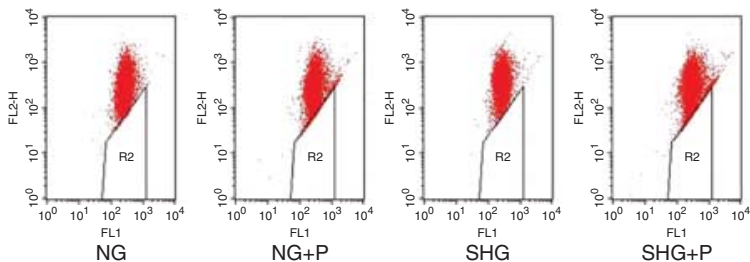

B

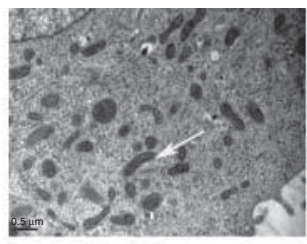

NG

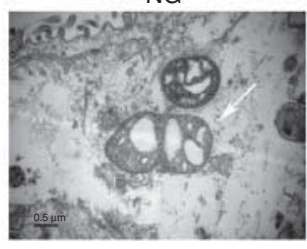

$\mathrm{SHG}+\mathrm{P}$

C
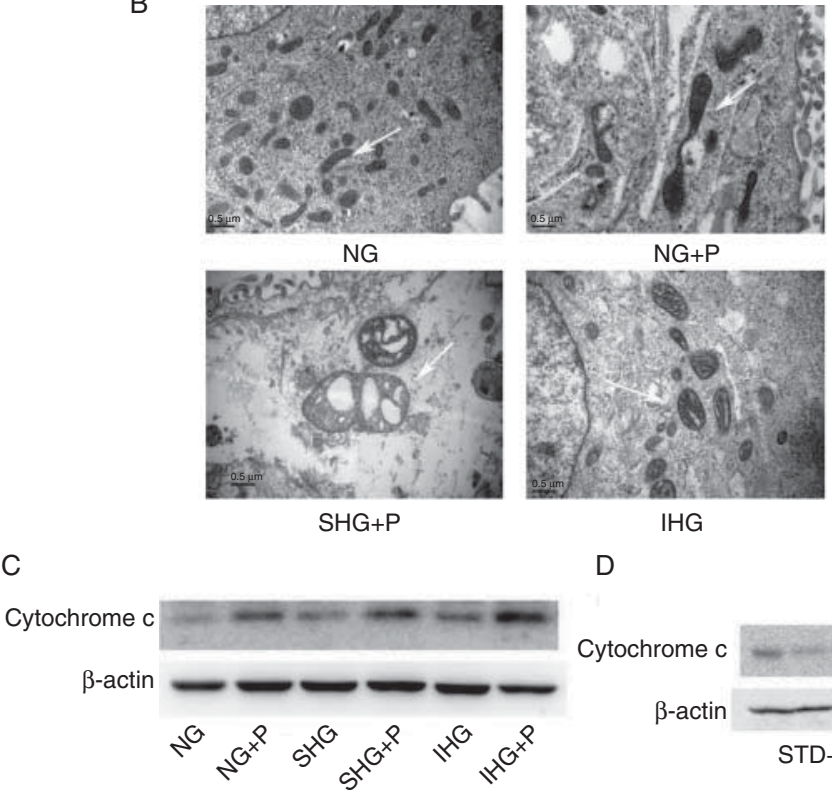

$\mathrm{NG}+\mathrm{P}$

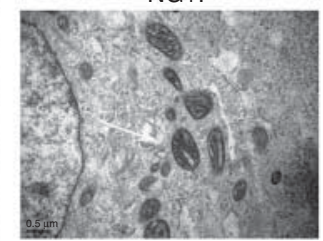

IHG

D

Cytochrome c

$\beta$-actin

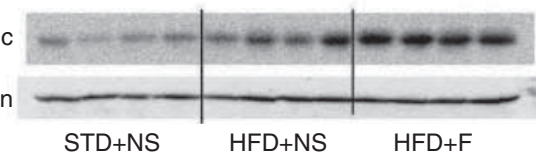

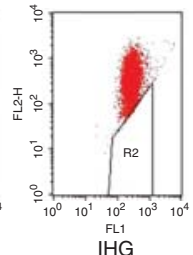
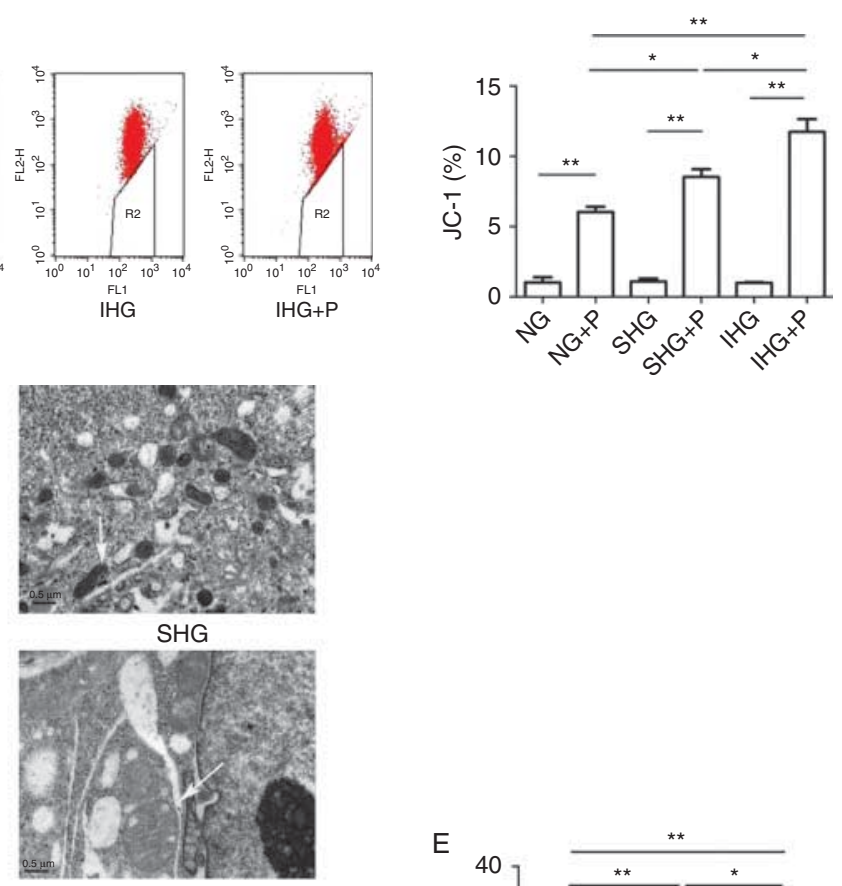

$\mathrm{IHG}+\mathrm{P}$

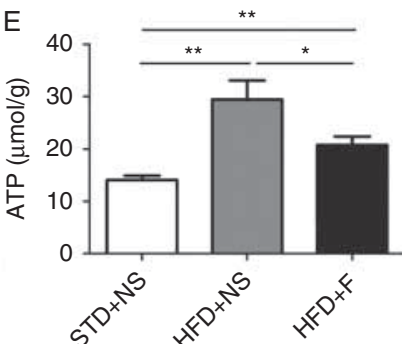

\section{Figure 6}

Effect of glucose fluctuation on hepatic mitochondrial dysfunction. Representative data obtained by JC- 1 fluorescence and flow cytometry to measure $\Delta \psi$ is presented $(\mathrm{A}) ; * P<0.05, * * P<0.01$, values are mean \pm s.E.M. of three replicates from three separate experiments. Representative electron micrographs showing mitochondrial ultrastructure are also presented ( $B$; arrows show mitochondria, $\times 30000$ magnification). Cytosolic cytochrome $\mathrm{c}$ expression was determined by western blotting in L02 cells (C) and mice (D) and normalized to the $\beta$-actin protein expression level. Hepatic ATP content was also determined in experimental mice (E). Values are means \pm s.E.M.; $n=10-12$ mice/group, ${ }^{*} P<0.05, * * P<0.01$.

\section{MPT inhibition reverses the apoptotic effect of glucose fluctuation}

The MPT is a critical factor in mitochondrial dysfunction and excess ROS production. To establish if this process was important in the observed increase in apoptosis following exposure to IHG and PA, we treated LO2 cells with the MPT inhibitor CsA. L02 cells pretreated with CsA had a reduced accumulation of JC-1 aggregates and a reduced release of cytochrome c following exposure to SHG and IHG in the presence of PA, compared to untreated cells (Fig. 7A and B). Furthermore, the ultrastructural abnormalities induced by IHG and PA were reversed by pretreatment with CsA (Fig. 7C), as was the increase in oxidative stress and the induction of LO2 cell apoptosis by IHG, SHG and NG in the presence of PA (Fig. 7D, E, F and G).

\section{Discussion}

The data presented herein provides evidence that IHG induces hepatocyte apoptosis and the corresponding reparative response under lipotoxicity in vitro and in vivo. Mechanistically, this increase in apoptosis is associated with increased ROS production and mitochondrial dysfunction, secondary to the induction of the MPT. These findings provide a possible mechanistic link between glucose fluctuation and the progression to NAFLD from simple steatosis to NASH.

Data from humans, as well as in vivo and in vitro experimental models, demonstrate that cell death, particularly apoptosis, is increased in NAFLD and NASH patients, suggesting that it is a crucial factor in disease progression (Feldstein \& Gores 2005, Machado \&

Published by Bioscientifica Ltd 
A

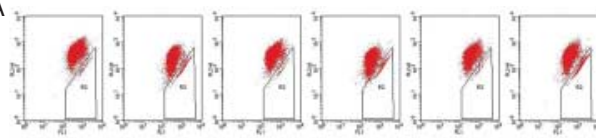

NG

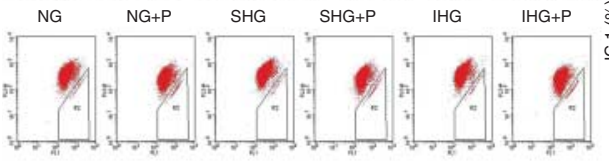

$\mathrm{NG}+\mathrm{CsA} \quad \mathrm{NG}+\mathrm{P}+\mathrm{Cs} \quad \mathrm{SHG}+\mathrm{CsA} \quad \mathrm{SHG}+\mathrm{P}+\mathrm{CsA} \quad \mathrm{IHG}+\mathrm{CsA} \quad \mathrm{IHG}+\mathrm{P}+\mathrm{CsA}$

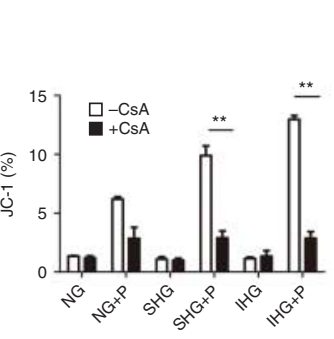

B

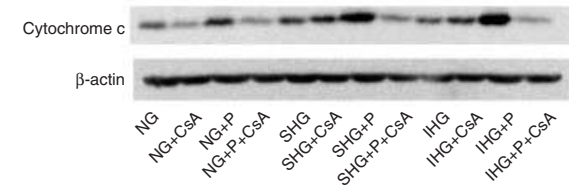

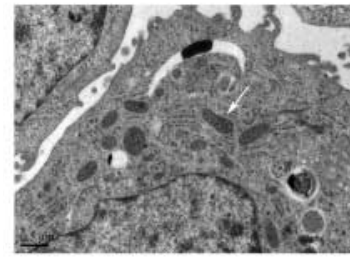

NG

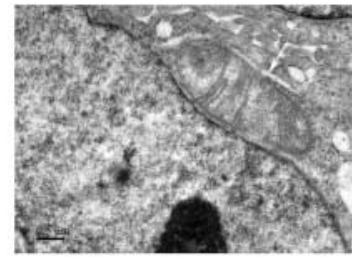

$\mathrm{IHG}+\mathrm{P}$

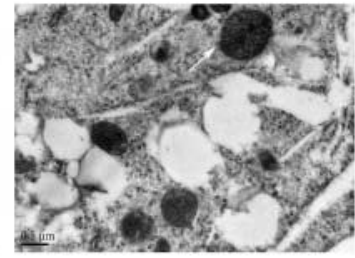

$\mathrm{IHG}+\mathrm{P}+\mathrm{CsA}$
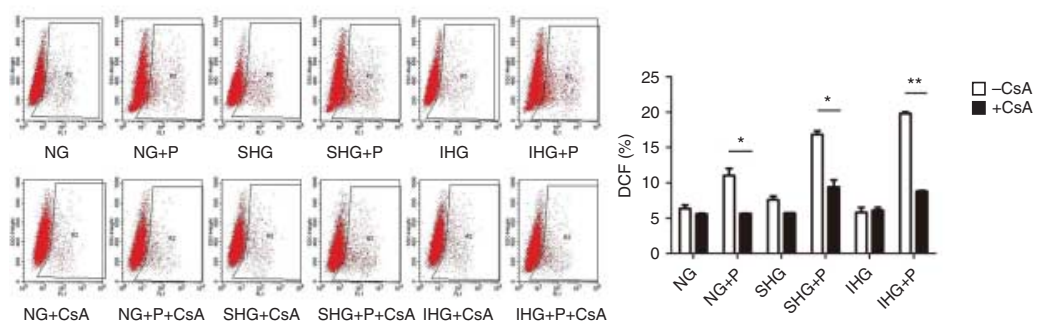

$\mathrm{E}$
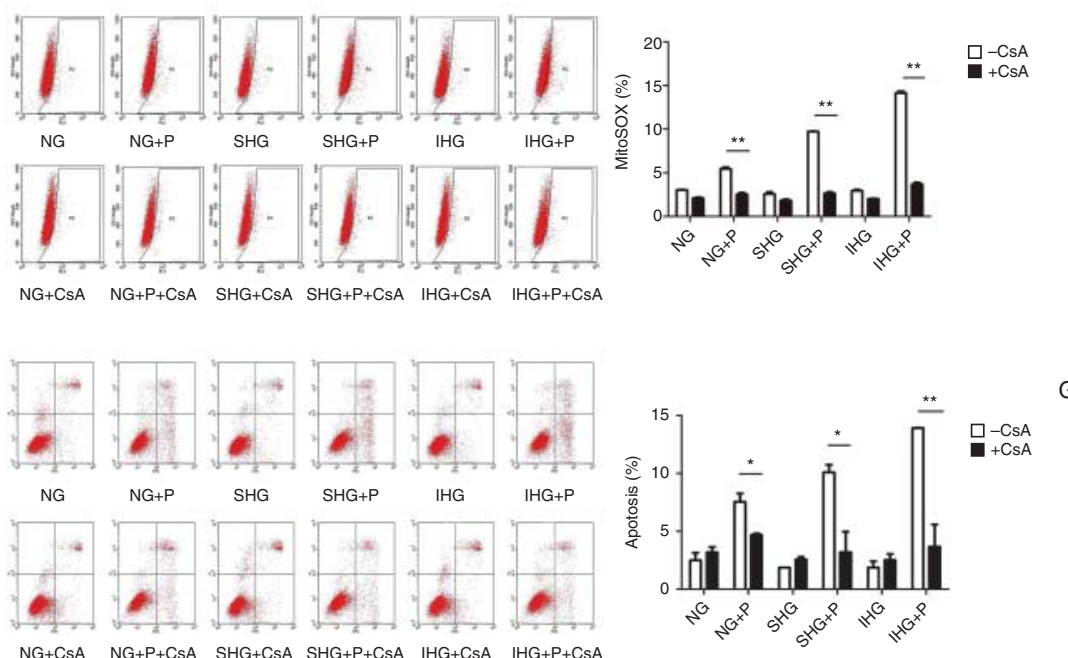

G

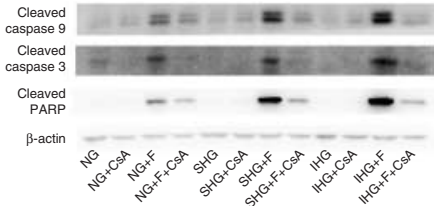

Figure 7

Effect of CsA on mitochondrial function, oxidative stress and hepatic apoptosis. Representative data showing the effect of CsA treatment on JC-1 fluorescence $(A)$, cytochrome $c$ release $(B)$ mitochondrial ultrastructure $(C)$, ROS production (D), mitochondrial superoxide (E), hepatic apoptosis (F)

Cortez-Pinto 2011). In our study, although no difference in the ALT level and hepatic lipid content were observed between mice fed a HFD injected with saline and glucose, mice injected with glucose did have evidence of increased and apoptosis-related proteins expression (G) in L02 cells under different experimental conditions are presented. ${ }^{\star} P<0.05, * * P<0.01$, values are mean \pm S.E.M. of three replicates from three separate experiments.

hepatocyte apoptosis. This link between IHG and apoptosis was further confirmed by exposing L02 cells to fluctuating glucose levels. Hepatocyte apoptosis triggers the activation of hepatic stellate cells to myofibroblasts,

Published by Bioscientifica Ltd. 
causes liver fibrosis and expands hepatic progenitor populations that produce chemoattractants to recruit various types of immune cells into the liver (Jou et al. 2008). Patients with T2DM and NAFLD show a significantly higher prevalence of NASH, fibrosis and cirrhosis than non-diabetic subjects with NAFLD (Leite et al. 2011, Smith \& Adams 2011, Doycheva et al. 2013). Furthermore, patients with severe vs mild fibrosis have been reported to have remarkably higher levels of glycemic variability (Hashiba et al. 2013). Our data confirms this association and shows that glucose fluctuation and lipotoxicity can significantly induce hepatocyte apoptosis, inflammation and fibrosis. Despite the increased levels of apoptosis observed under conditions of IHG, no differences were observed in markers of glucose levels, lipid deposition and liver enzyme levels in HFD-induced obese mice. Further study will be required to determine if this was due to the relatively short observation period in the current study or to the differences in the frequency and amplitude of glucose fluctuations in animal models vs diabetic patients.

An important factor in the progression of NAFLD is oxidative stress, with NAFLD patients being reported to have increased oxidative stress (Sumida et al. 2003, Yesilova et al. 2005) and a decrease in antioxidant defenses (Videla et al. 2004). T2DM is also characterized by the presence of oxidative stress (Davi et al. 1999), and diabetic patients with NAFLD have higher levels of markers of oxidative stress compared to diabetic patients without NAFLD (Narasimhan et al. 2010). Recently, several studies have demonstrated that IHG can generate more ROS than SHG in endothelial cells (Quagliaro et al. 2003, Piconi et al. 2006), islet cell (Del Guerra et al. 2007, Kim et al. 2012) and Schwann cells (Sun et al. 2012a,b). We found that in the presence of lipotoxicity, SHG produced an increase in oxidative stress; moreover, the IHG appeared to further enhance oxidative stress. Previous studies in HepG2 cells demonstrated that ROS generation is increased in cells cultured in $30 \mathrm{mmol} / 1$ glucose for $48 \mathrm{~h}$, but with no changes in cell viability until 7 days (Palmeira et al. 2007). It was reported that HepG2 cells cultured in $50 \mathrm{mmol} / \mathrm{l}$ glucose for $72 \mathrm{~h}$ showed a significant increase in apoptosis, accompanied by an increase in ROS levels (Chandrasekaran et al. 2010). Different cell models adopted or the shorter incubation period or the lower glucose concentration could possibly explain the discrepancy in these studies. Here, increased oxidative stress and hepatocyte apoptosis was only observed in the context of lipotoxicity. The concern is that lipotoxicity is quite common in T2DM, thus it could not only initiate the development of NAFLD but also promote its progression.
Such an effect would further be enhanced in the presence of high glucose levels, especially IHG. Thus, lipotoxicity and IHG might be an important mechanism mediating the progression of NAFLD to NASH in T2DM.

It is well recognized that mitochondria play a critical role in the induction of cellular oxidative stress and apoptosis, with the most recent attention focused on the role of the MPT in this process (Lee \& Wei 2000). It has been proposed that the induction of the MPT itself can increase production of ROS from the respiratory chain (Zamzami et al. 1995) through the release of mitochondrial cytochrome $\mathrm{c}$ that then impairs transfer of electrons. In turn, this increase may cause oxidation of critical thiol sites in the MPT pore (Lemasters et al. 1998) causing further induction of MPT with the subsequent release of cytochrome $\mathrm{c}$ and other apoptosis-inducing proteins. Thus, a vicious cycle in the mitochondria originally stimulated by oxidative stress may further perpetuate and magnify the intracellular oxidative stress. Here, the MPT inhibitor CsA prevented PA-induced mitochondrial dysfunction, oxidative stress and hepatocyte apoptosis, indicating that the opening of the MPT pore is an essential prerequisite for triggering the apoptosis. IHG exacerbated this vicious cycle of MPT-ROS production in the mitochondria, leading to excess liver cell apoptosis.

In summary, our findings support the concept that glucose fluctuation accelerates hepatocyte apoptosis, inflammation and fibrosis in the context of lipotoxicity by increasing oxidative stress and mitochondrial dysfunction. Furthermore, the MPT seems to play a critical role in the initiation of hepatocyte apoptosis caused by lipotoxicity in conjunction with SHG and IHG, both of which are common in T2DM. We conclude that a better control of mean glucose levels and glucose fluctuations may be of potential benefit in slowing the progression of NAFLD in T2DM.

\section{Supplementary data}

This is linked to the online version of the paper at http://dx.doi.org/10.1530/ JME-15-0101.

\section{Declaration of interest}

The authors declare that there is no conflict of interest that could be perceived as prejudicing the impartiality of the research reported.

\section{Funding}

This work was supported by the National Natural Science Foundation of China, Nos 81000364, 81270924 and 81471099.

Published by Bioscientifica Ltd. 


\section{Acknowledgements}

The authors are grateful for the technical expertise of Hu Xiaotong (Key Laboratory of Biotherapy of Zhejiang Province, Sir Run Run Shaw Hospital, Zhejiang University, School of Medicine, Hangzhou, Zhejiang, China).

\section{References}

Adams LA \& Angulo P 2005 Recent concepts in non-alcoholic fatty liver disease. Diabetic Medicine 22 1129-1133. (doi:10.1111/j.1464-5491. 2005.01748.x)

Argo CK, Northup PG, Al-Osaimi AM \& Caldwell SH 2009 Systematic review of risk factors for fibrosis progression in non-alcoholic steatohepatitis. Journal of Hepatology 51 371-379. (doi:10.1016/j.jhep. 2009.03.019)

Bellentani S \& Marino M 2009 Epidemiology and natural history of nonalcoholic fatty liver disease (NAFLD). Annals of Hepatology $\mathbf{8}$ (Suppl 1) S4-S8.

Chandrasekaran K, Swaminathan K, Chatterjee S \& Dey A 2010 Apoptosis in HepG2 cells exposed to high glucose. Toxicology in Vitro 24 387-396. (doi:10.1016/j.tiv.2009.10.020)

Davi G, Ciabattoni G, Consoli A, Mezzetti A, Falco A, Santarone S, Pennese E, Vitacolonna E, Bucciarelli T, Costantini F et al. 1999 In vivo formation of 8 -iso-prostaglandin $\mathrm{f} 2 \alpha$ and platelet activation in diabetes mellitus: effects of improved metabolic control and vitamin E supplementation. Circulation 99 224-229. (doi:10.1161/01.CIR.99.2.224)

Day CP \& James OF 1998 Steatohepatitis: a tale of two "hits"? Gastroenterology 114 842-845. (doi:10.1016/S0016-5085(98)70599-2)

Del Guerra S, Grupillo M, Masini M, Lupi R, Bugliani M, Torri S, Boggi U, Del Chiaro M, Vistoli F, Mosca F et al. 2007 Gliclazide protects human islet $\beta$-cells from apoptosis induced by intermittent high glucose. Diabetes/Metabolism Research and Reviews 23 234-238. (doi:10.1002/ dmrr.680)

Doycheva I, Patel N, Peterson M \& Loomba R 2013 Prognostic implication of liver histology in patients with nonalcoholic fatty liver disease in diabetes. Journal of Diabetes and its Complications 27 293-300. (doi:10.1016/j.jdiacomp.2012.10.008)

Feldstein AE \& Gores GJ 2005 Apoptosis in alcoholic and nonalcoholic steatohepatitis. Frontiers in Bioscience 10 3093-3099. (doi:10.2741/ 1765)

Feldstein AE, Werneburg NW, Canbay A, Guicciardi ME, Bronk SF, Rydzewski R, Burgart LJ \& Gores GJ 2004 Free fatty acids promote hepatic lipotoxicity by stimulating TNF- $\alpha$ expression via a lysosomal pathway. Hepatology 40 185-194. (doi:10.1002/hep.20283)

Halestrap AP \& Brenner C 2003 The adenine nucleotide translocase: a central component of the mitochondrial permeability transition pore and key player in cell death. Current Medicinal Chemistry 10 1507-1525. (doi:10.2174/0929867033457278)

Halestrap AP, Connern CP, Griffiths EJ \& Kerr PM 1997 Cyclosporin A binding to mitochondrial cyclophilin inhibits the permeability transition pore and protects hearts from ischaemia/reperfusion injury. Molecular and Cellular Biochemistry 174 167-172. (doi:10.1023/ A:1006879618176)

Hashiba M, Ono M, Hyogo H, Ikeda Y, Masuda K, Yoshioka R, Ishikawa Y, Nagata Y, Munekage K, Ochi T et al. 2013 Glycemic variability is an independent predictive factor for development of hepatic fibrosis in nonalcoholic Fatty liver disease. PLoS ONE 8 e76161. (doi:10.1371/ journal.pone.0076161)

Jou J, Choi SS \& Diehl AM 2008 Mechanisms of disease progression in nonalcoholic fatty liver disease. Seminars in Liver Disease 28 370-379. (doi:10.1055/s-0028-1091981)

Kim M, Chung H, Yoon C, Lee E, Kim T, Kwon M, Lee S, Rhee B \& Park J 2012 Increase of INS-1 cell apoptosis under glucose fluctuation and the involvement of FOXO-SIRT pathway. Diabetes Research and Clinical Practice 98 132-139. (doi:10.1016/j.diabres.2012.04.013)
Kroemer G \& Reed JC 2000 Mitochondrial control of cell death. Nature Medicine 6 513-519. (doi:10.1038/74994)

Lee HC \& Wei YH 2000 Mitochondrial role in life and death of the cell. Journal of Biomedical Science 7 2-15. (doi:10.1007/BF02255913)

Leite NC, Salles GF, Araujo AL, Villela-Nogueira CA \& Cardoso CR 2009 Prevalence and associated factors of non-alcoholic fatty liver disease in patients with type-2 diabetes mellitus. Liver International 29 113-119. (doi:10.1111/j.1478-3231.2008.01718.x)

Leite NC, Villela-Nogueira CA, Pannain VL, Bottino AC, Rezende GF, Cardoso CR \& Salles GF 2011 Histopathological stages of nonalcoholic fatty liver disease in type 2 diabetes: prevalences and correlated factors. Liver International 31 700-706. (doi:10.1111/j.1478-3231.2011.02482.x)

Lemasters JJ, Nieminen AL, Qian T, Trost LC, Elmore SP, Nishimura Y, Crowe RA, Cascio WE, Bradham CA, Brenner DA et al. 1998 The mitochondrial permeability transition in cell death: a common mechanism in necrosis, apoptosis and autophagy. Biochimica et Biophysica Acta 1366 177-196. (doi:10.1016/S0005-2728(98)00112-1)

Livak KJ \& Schmittgen TD 2001 Analysis of relative gene expression data using real-time quantitative PCR and the $2(-\Delta \Delta C(\mathrm{~T}))$ method. Methods 25 402-408. (doi:10.1006/meth.2001.1262)

Machado MV \& Cortez-Pinto H 2011 Cell death and nonalcoholic steatohepatitis: where is ballooning relevant? Expert Review of Gastroenterology \& Hepatology 5 213-222. (doi:10.1586/egh.11.16)

Malhi H \& Gores GJ 2008 Cellular and molecular mechanisms of liver injury. Gastroenterology 134 1641-1654. (doi:10.1053/j.gastro. 2008.03.002)

Monnier L, Mas E, Ginet C, Michel F, Villon L, Cristol JP \& Colette C 2006 Activation of oxidative stress by acute glucose fluctuations compared with sustained chronic hyperglycemia in patients with type 2 diabetes. Journal of the American Medical Association 295 1681-1687. (doi:10. 1001/jama.295.14.1681)

Narasimhan S, Gokulakrishnan K, Sampathkumar R, Farooq S, Ravikumar R, Mohan V \& Balasubramanyam M 2010 Oxidative stress is independently associated with non-alcoholic fatty liver disease (NAFLD) in subjects with and without type 2 diabetes. Clinical Biochemistry 43 815-821. (doi:10.1016/j.clinbiochem.2010.04.003)

Palmeira CM, Rolo AP, Berthiaume J, Bjork JA \& Wallace KB 2007 Hyperglycemia decreases mitochondrial function: the regulatory role of mitochondrial biogenesis. Toxicology and Applied Pharmacology 225 214-220. (doi:10.1016/j.taap.2007.07.015)

Piconi L, Quagliaro L, Assaloni R, Da Ros R, Maier A, Zuodar G \& Ceriello A 2006 Constant and intermittent high glucose enhances endothelial cell apoptosis through mitochondrial superoxide overproduction. Diabetes/Metabolism Research and Reviews 22 198-203. (doi:10.1002/ dmrr.613)

Prashanth M, Ganesh HK, Vima MV, John M, Bandgar T, Joshi SR, Shah SR, Rathi PM, Joshi AS, Thakkar H et al. 2009 Prevalence of nonalcoholic fatty liver disease in patients with type 2 diabetes mellitus. Journal of the Association of Physicians of India $\mathbf{5 7}$ 205-210.

Quagliaro L, Piconi L, Assaloni R, Martinelli L, Motz E \& Ceriello A 2003 Intermittent high glucose enhances apoptosis related to oxidative stress in human umbilical vein endothelial cells: the role of protein kinase C and NAD(P)H-oxidase activation. Diabetes 52 2795-2804. (doi:10.2337/ diabetes.52.11.2795)

Quagliaro L, Piconi L, Assaloni R, Da Ros R, Maier A, Zuodar G \& Ceriello A 2005 Intermittent high glucose enhances ICAM-1, VCAM-1 and E-selectin expression in human umbilical vein endothelial cells in culture: the distinct role of protein kinase $\mathrm{C}$ and mitochondrial superoxide production. Atherosclerosis 183 259-267. (doi:10.1016/ j.atherosclerosis.2005.03.015)

Russmann S, Kullak-Ublick GA \& Grattagliano I 2009 Current concepts of mechanisms in drug-induced hepatotoxicity. Current Medicinal Chemistry 16 3041-3053. (doi:10.2174/092986709788803097)

Salvioli S, Ardizzoni A, Franceschi C \& Cossarizza A 1997 JC-1, but not DiOC6(3) or rhodamine 123, is a reliable fluorescent probe to assess delta psi changes in intact cells: implications for studies on 
mitochondrial functionality during apoptosis. FEBS Letters 411 77-82. (doi:10.1016/S0014-5793(97)00669-8)

Shi H, Kokoeva MV, Inouye K, Tzameli I, Yin H \& Flier JS 2006 TLR4 links innate immunity and fatty acid-induced insulin resistance. Journal of Clinical Investigation 116 3015-3025. (doi:10.1172/JCI28898)

Smith BW \& Adams LA 2011 Nonalcoholic fatty liver disease and diabetes mellitus: pathogenesis and treatment. Nature Reviews. Endocrinology $\mathbf{7}$ 456-465. (doi:10.1038/nrendo.2011.72)

Sumida Y, Nakashima T, Yoh T, Furutani M, Hirohama A, Kakisaka Y, Nakajima Y, Ishikawa H, Mitsuyoshi H, Okanoue T et al. 2003 Serum thioredoxin levels as a predictor of steatohepatitis in patients with nonalcoholic fatty liver disease. Journal of Hepatology 38 32-38. (doi:10.1016/S0168-8278(02)00331-8)

Sun LQ, Chen YY, Wang X, Li XJ, Xue B, Qu L, Zhang TT, Mu YM \& Lu JM $2012 a$ The protective effect of $\alpha$ lipoic acid on Schwann cells exposed to constant or intermittent high glucose. Biochemical Pharmacology $\mathbf{8 4}$ 961-973. (doi:10.1016/j.bcp.2012.07.005)

Sun LQ, Xue B, Li XJ, Wang X, Qu L, Zhang TT, Zhao J, Wang BA, Zou XM, Mu YM et al. 2012b Inhibitory effects of salvianolic acid B on apoptosis of Schwann cells and its mechanism induced by intermittent high glucose. Life Sciences 90 99-108. (doi:10.1016/j.lfs.2011.10.001)

Targher G, Bertolini L, Padovani R, Rodella S, Tessari R, Zenari L, Day C \& Arcaro G 2007 Prevalence of nonalcoholic fatty liver disease and its association with cardiovascular disease among type 2 diabetic patients. Diabetes Care 30 1212-1218. (doi:10.2337/dc06-2247)

Unger RH \& Orci L 2002 Lipoapoptosis: its mechanism and its diseases. Biochimica et Biophysica Acta 1585 202-212. (doi:10.1016/ S1388-1981(02)00342-6)

Videla LA, Rodrigo R, Orellana M, Fernandez V, Tapia G, Quinones L, Varela N, Contreras J, Lazarte R, Csendes A et al. 2004 Oxidative stress-related parameters in the liver of non-alcoholic fatty liver disease patients. Clinical Science 106 261-268. (doi:10.1042/CS20030285)

Wang Z, Li L, Zheng F, Jia C, Ruan Y \& Li H 2011 Correlation between the amplitude of glucose excursion and the oxidative/antioxidative system in subjects with different types of glucose regulation. Biomedical and Environmental Sciences 24 68-73. (doi:10.3967/0895-3988.2011.01.009)

Yamada F, Saito T, Abe T, Tsuchiya T, Sato Y, Kenjo A, Kimura T \& Gotoh M 2007 Ischemic preconditioning enhances regenerative capacity of hepatocytes in long-term ischemically damaged rat livers. Journal of Gastroenterology and Hepatology 22 1971-1977. (doi:10.1111/j.14401746.2006.04711.x)

Yesilova Z, Yaman H, Oktenli C, Ozcan A, Uygun A, Cakir E, Sanisoglu SY, Erdil A, Ates Y, Aslan M et al. 2005 Systemic markers of lipid peroxidation and antioxidants in patients with nonalcoholic Fatty liver disease. American Journal of Gastroenterology 100 850-855. (doi:10.1111/ j.1572-0241.2005.41500.x)

Zamzami N, Marchetti P, Castedo M, Decaudin D, Macho A, Hirsch T, Susin SA, Petit PX, Mignotte B \& Kroemer G 1995 Sequential reduction of mitochondrial transmembrane potential and generation of reactive oxygen species in early programmed cell death. Journal of Experimental Medicine 182 367-377. (doi:10.1084/jem.182.2.367)

Zheng F, Lu W, Jia C, Li H, Wang Z \& Jia W 2010 Relationships between glucose excursion and the activation of oxidative stress in patients with newly diagnosed type 2 diabetes or impaired glucose regulation. Endocrine 37 201-208. (doi:10.1007/s12020-009-9296-6)

Zorov DB, Filburn CR, Klotz LO, Zweier JL \& Sollott SJ 2000 Reactive oxygen species (ROS)-induced ROS release: a new phenomenon accompanying induction of the mitochondrial permeability transition in cardiac myocytes. Journal of Experimental Medicine 192 1001-1014. (doi:10.1084/jem.192.7.1001)

Received in final form 22 August 2015 Accepted 24 August 2015
(C) 2015 Society for Endocrinology Printed in Great Britain 